\title{
Dietary exosome-miR-23b may be a novel therapeutic measure for preventing Kashin-Beck disease (Review)
}

\author{
YUJIE NING ${ }^{*}$, XI WANG ${ }^{*}$, PAN ZHANG, AMIN LIU, XIN QI, MEIDAN LIU and XIONG GUO \\ Institute of Endemic Diseases, School of Public Health, Xi'an Jiaotong University Health Science Center, Key Laboratory of \\ Trace Elements and Endemic Diseases, National Health and Family Planning Commission, Xi'an, Shaanxi 710061, P.R. China
}

Received October 7, 2017; Accepted January 4, 2018

DOI: $10.3892 /$ etm.2018.5885

\begin{abstract}
Previous studies have identified a close association between diet and the prevalence of Kashin-Beck disease (KBD); however, the mechanisms via which the diet protects against KBD-associated cartilage injury has remained elusive. Recent international research studies have revealed a therapeutic role of dietary exosome micro (mi)RNAs in repairing chondrocyte lesions by regulating genes and proteins associated with cellular apoptosis and extracellular matrix. Vital molecules affecting bio-functions of chondrocytes, including miR-23b and protein kinase cyclic AMP-activated catalytic subunit $\beta$, were preliminarily identified to be dysregulated in cells and cartilage tissue of KBD patients. The function of dietary exosome in the repair of chondrocyte lesions in KBD is a novel topic in this field. It is worth exploring the protective role of dietary exosome-miR-23b against chondrocyte damage through the regulation of the protein kinase A (PKA) signaling pathway. The following aims are significant in future studies: i) To verify the association between exosome and cartilage damage in KBD patients; ii) to identify whether the protective mechanism of miR-23b in cartilage damage proceeds through regulating the PKA pathway; and iii) to explore the therapeutic role of dietary exosome-miR-23b in repairing chondrocyte lesions induced by environmental risk factors. These ideas may help establish the therapeutic role and mechanisms of dietary exosome-miR-23b in repairing chondrocyte lesions at the molecular, cellular and organismal level. These studies may simultaneously elucidate the disease
\end{abstract}

Correspondence to: Professor Xiong Guo, Institute of Endemic Diseases, School of Public Health, Xi'an Jiaotong University Health Science Center, Key Laboratory of Trace Elements and Endemic Diseases, National Health and Family Planning Commission, 76 Yanta West Road, Xi'an, Shaanxi 710061, P.R. China

E-mail: guox@xjtu.edu.cn

*Contributed equally

Key words: exosome, microRNA-23b, Kashin-Beck disease, diet, environmental risk factor pathogenesis and provide evidence for novel biomarkers and therapeutic methods for KBD.

\section{Contents}

1. Introduction

2. Dietary changes in endemic areas are closely associated with the reduction of the prevalence of KBD

3. The exosome, an important carrier of intercellular information, has a vital part in the etiology and pathology of osteoarthropathy and may be studied as a therapeutic strategy

4. Regulation of the PKA signaling pathway in chondrocytes by miR-23b may be involved in the mechanism of cartilage damage in KBD

5. Dietary exosomal miRNAs are resistant to digestion and exert functions across species

6. Potential protective effect of dietary exosome-miR-23b in residents of endemic regions and KBD patients from articular cartilage injury stimulated by environmental risk factors through regulation of the PKA pathway

7. Conclusions

\section{Introduction}

Kashin-Beck disease (KBD) is a type of endemic osteoarthropathy. It is characterized by onset during childhood, deformed phalangeal joints or even limbs and degenerative cartilage damage (1). China has the broadest endemic areas, the highest number of patients affected and the highest prevalence of KBD worldwide. According the 2016 China Health Statistical Yearbook, a total of 567,600 patients, including 12,730 juvenile patients (age, $\leq 13$ years) in 378 counties, and $>104$ million residents in China are currently at risk (http://www.nhfpc.gov.cn). Furthermore, new cases were recently diagnosed in Tibet. The X-ray-positive rate of phalangeal damage in children, which indicates new KBD cases, was $6.67 \%$ in Re-yu Village of Bian-ba county in Tibet (2).

Identified environmental risk factors for KBD are prevalent in China. Selenium supplementation has no longer been applied nationwide since 2012, so that environmental selenium 
is still low in KBD endemic areas. Mycotoxin contamination in food is detected in Qinghai Province, a severely endemic region (3). Therefore, the threat of KBD in Chinese populations persists. 'Focus on prevention and control of endemic diseases' is one of the most important tasks stated in the 13th Five-Year Plan for health and wellness of the public in China (http://www.moh.gov.cn/). Hence, there is an urgent demand to identify the underlying molecular mechanisms of articular damage in patients induced by environmental risk factors, and to develop effective prevention and treatment measures against these risk factors to 'eliminate the hazard of KBD', as proposed in the plan.

\section{Dietary changes in endemic areas are closely associated with the reduction of the prevalence of KBD}

Despite the elusive etiology and pathology of KBD after $>160$ years of investigation, various hypotheses have been proposed by experts worldwide. The hypotheses commonly involve environmental factors and may be summarized into two major models. One view is that the occurrence of KBD is associated with a specific geographical and ecological environment. The model suggests that the overabundance, deficiency or disproportion of certain elements in the endemic environment may cause an abnormal nutritional status of certain elements (e.g., selenium deficiency) in the body through the food chain, which then gradually induces metabolic irregularities followed by disorders in physiological function, and ultimately disease (4-7). The other view suggests that food contamination is the primary cause of KBD. It holds that mycotoxin contamination (e.g., T-2 toxin) of locally produced cereals and organic poisoning (e.g., humus) in drinking water may increase the levels of reactive oxygen species and free radicals in the body, which may damage chondrocytes, disturb the extracellular matrix and induce excessive apoptosis and necrosis of chondrocytes in KBD patients $(8,9)$.

The above hypotheses suggest that diet is significantly associated with KBD. A number of epidemiological investigations, pedigree studies and sib-pair studies have indicated that KBD exhibits a familial aggregation but no familial heredity $(10,11)$. The incidence of KBD and the severity of the disease were determined to be positively correlated with the long-term intake of locally produced cereals (e.g., corn and wheat), and drinking water (e.g., ditch and cellar water) polluted by organic complexes (e.g., humic acid) (12). Low selenium in the environment tends to cause low nutritional selenium in the body through the food chain, which may increase the risk of KBD; by contrast, selenium supplementation, e.g., through selenium-enriched salt, effectively decreased the incidence of KBD in children, and may delay or prevent articular cartilage lesions in patients (13). Long-term consumption of cereals contaminated with mycotoxin (e.g., T-2 toxin) was reported to significantly elevate the risk of KBD (14); furthermore, low selenium combined with T-2 toxin induced human-KBD-like cartilage damage in rats as determined via pathological, biochemical and molecular biology analyses (15). Finally, a cross-sectional study on nutrient intake of children aged 4-14 years from Lin-you and Yong-shou counties, which are severely endemic areas, reported that the intake proportion of cereal products was high, while the intake of meat, eggs and bean products was low; furthermore, inadequate intake of proteins, minerals and vitamins compared with the Estimated Average Requirement in China was identified $(16,17)$. This evidence suggested that an undiversified diet structure and insufficient nutrient intake may promote the occurrence of KBD.

Based on the above results, multivariate regression analyses indicated that the major risk factors for KBD are low nutritional selenium, insufficient consumption of bean products (shortage of proteins), poor storage conditions for cereals, and food contamination by mycotoxin (18). In recent decades, comprehensive measures have been established to prevent KBD, including change of cereal source, improvement of food storage conditions, diversified diet structure, grain for green and relocation $(19,20)$. The incidence and severity of KBD have exhibited yearly decreases. However, the mechanisms by which the diet affects the pathogenesis of KBD remains elusive, and it is worth further investigating them in order to develop novel, effective and accessible methods for preventing KBD.

\section{The exosome, an important carrier of intercellular information, has a vital part in the etiology and pathology of osteoarthropathy and may be studied as a therapeutic strategy}

Exosomes are a type of extracellular vesicle. The prospect of clinical applications of exosomes in various fields has been postulated in 2016 (21). Exosomes widely exist in animals, plants and microbes, e.g., in bodily fluids (synovial fluid, blood, urine, saliva and milk) and the supernatant of cell cultures. Exosomes have a 40-100 nm bilayer membrane structure formed by endocytosis, and they contain various bioactive substances, including miRNAs, mRNAs, proteins and lipids (22). These molecules are termed 'cargo' and are released into the intercellular space by exocytosis in response to the body's requirements or pathogen stimulation. Adjacent or long-distance cells may selectively absorb the released substances by endocytosis for use in cellular processes, including proliferation, differentiation, migration, apoptosis and extracellular matrix degradation (Fig. 1) (23). The composition of cargo affects the health status of organisms and vice versa. Changes in cargo composition may induce abnormal expression of genes and proteins in the body. In addition, psychological and/or physiological disorders may lead to abnormal cargo composition in exosomes (24).

Kato et al (25) and Withrow et al (26) reported that exosome and cargo concentrations were abnormal in osteoarthropathy, as reflected by differential expression of genes associated with cell apoptosis, inflammatory factors and extracellular matrix. In 2016, a pathogenesis study of osteoarthritis (OA) indicated that exosomes, extracted from chondrocytes cultured with interleukin (IL)-1 $\beta$, increased the level of matrix metalloproteinase (MMP)-13 in fibroblast-like synoviocytes up to 3-fold, accompanied by elevated levels of IL-1 $\beta$, tumor necrosis factor (TNF)- $\alpha$ and cyclooxygenase-2 (26). It suggests that exosomes of chondrocytes affect the expression of inflammatory factors and MMP, which further causes metabolic disorders of the cartilage matrix and induces pathological changes. 


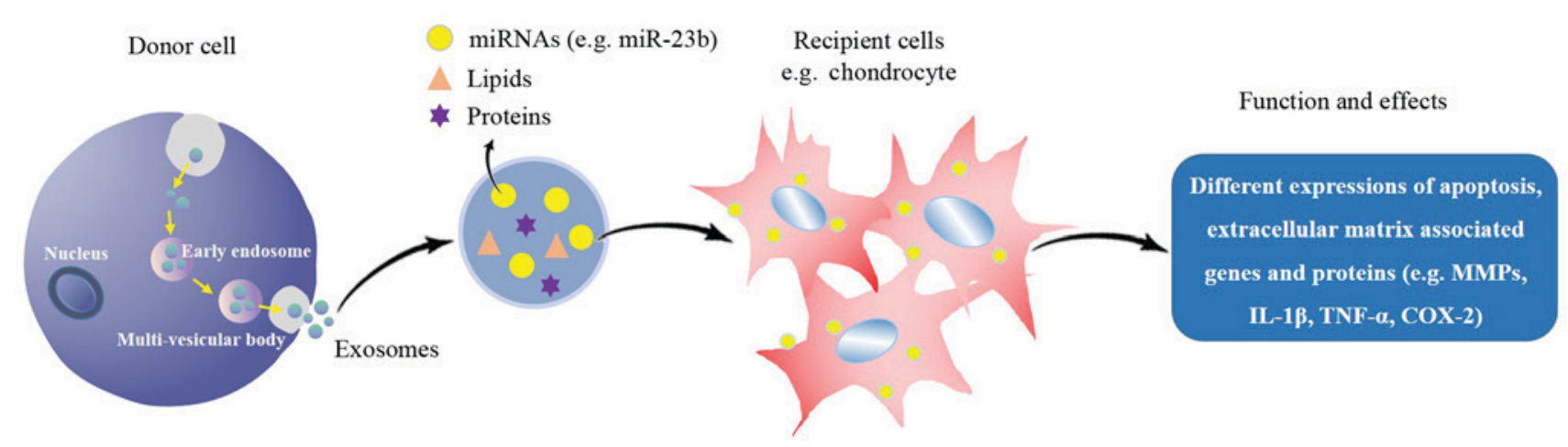

Figure 1. Process of exosomes delivering substances from donor cells to recipient cells. MMP, matrix metalloproteinase; IL, interleukin; TNF, tumor necrosis factor; $\mathrm{COX}$, cyclooxygenase.

Exosomes also have an important role in the development of rheumatoid arthritis (RA) by influencing the synthesis, transportation and activation of disease-associated components, including immune complexes, micro (mi)RNA, inflammatory factors and proteases (26). In an animal model of RA, extracellular matrix degradation was induced by exosomal cargo, including MMPs, a disintegrin and metalloproteinase with thrombospondin motifs-5, hexosaminidase D and B-glucuronidase, released by inflammatory cytokines, and resulting in cartilage damage and aggravated inflammatory reaction (23).

The amount and activity of IL- $1 \beta$ and TNF- $\alpha$ in the chondrocytes, serum and synovial fluid of KBD patients were reported to be significantly increased compared with those in normal controls. Overexpression of MMP-13 was also detected in articular cartilage of KBD patients, in addition to substantial loss of type II collagen and aggrecan (27). To date, it has not been explored whether the pathological changes in KBD described above are associated with abnormal exosomes. Furthermore, whether the level of exosomal cargo may be affected by low selenium and T-2 toxin is also a novel topic in this field.

As traditional therapies for diseases comprising cartilage injury, including KBD and OA, intra-articular injection of sodium hyaluronate may be performed to relieve pain and arthroplasty may be applied to regain working capacity for advanced patients. However, these therapies are limited by utility duration and side-effects and are not able to decrease the incidence of the disease. Exosomal cargo (e.g., miRNAs) derived from autologous cells, when used for therapeutic purposes, may contribute to the normal biological function without side effects, including immunogenicity and tumourigenicity. Exosomal miRNA-140-5p derived from human synovial mesenchymal stem cells (hSMSCs) promoted the proliferation and migration of chondrocytes, and also depressed the inhibition of extracellular matrix molecules, including type II collagen and aggrecan $(28,29)$. Exosomes derived from hSMSCs are also able to promote osteochondral regeneration $(30,31)$. Due to these effects, the maintenance of cartilage homeostasis was facilitated and tissue regeneration was enhanced, and exosomes may therefore be an effective means to prevent OA.

Therefore, based on the therapeutic role of exosomes in preventing cartilage injury-associated diseases, identification of the link between exosomes and the pathogenesis of KBD is necessary for the etiological study and development of novel prevention measures for KBD.

\section{Regulation of the PKA signaling pathway in chondrocytes by miR-23b may be involved in the mechanism of cartilage damage in $\mathrm{KBD}$}

miRs are small, non-coding RNAs consisting of 20-24 nucleotides, which regulate gene expression in humans and other species (32). miR-23b has recently been identified to be associated with osteoarticular diseases. It is involved in several cellular functions, including proliferation, differentiation, reconstruction of the cytoskeleton and migration. miR-23b was identified to promote the differentiation of human mesenchymal stem cells (hMSCs) into chondrocytes (33). It also depressed the expression of Smad3 at the gene and protein level, which ameliorated lipopolysaccharide-induced inhibition of osteogenic differentiation in MC3T3-E1 pre-osteoblast cells (34).

Protein kinase A (PKA) is composed of two regulatory subunits and two catalytic subunits and is normally inactive. PKA is activated by the combination of cyclic AMP (cAMP) and its regulatory subunits, and it is also known as cAMP-dependent protein kinase (35). The activated form is functional in numerous biological processes by phosphorylating target proteins, including those involved in the regulation of glycogen, sugar and lipid metabolism (36). Prostaglandin D2 dose-dependently decreased the IL-1 $\beta$-induced production of MMP-1 and MMP-13 protein and mRNA in chondrocytes through the PKA signaling pathway. This suggests that the PKA may be a promising therapeutic target for the prevention of cartilage degradation $(37,38)$. In addition, Yokoyama et al (39) reported that the PKA pathway is involved in the proliferation of chondrocytes in patients with neonatal-onset multisystem inflammatory disease.

Interactions between miR-23b and the PKA pathway have a role in maintaining normal physiological functions of chondrocytes. miR-23b may promote the production of aggrecan, SRY-box 9, as well as type II and type X collagens, and it also suppresses the productions of MMPs. More importantly, it affects the expression of phospho-cAMP response element binding protein, a key phosphorylated response element, to 

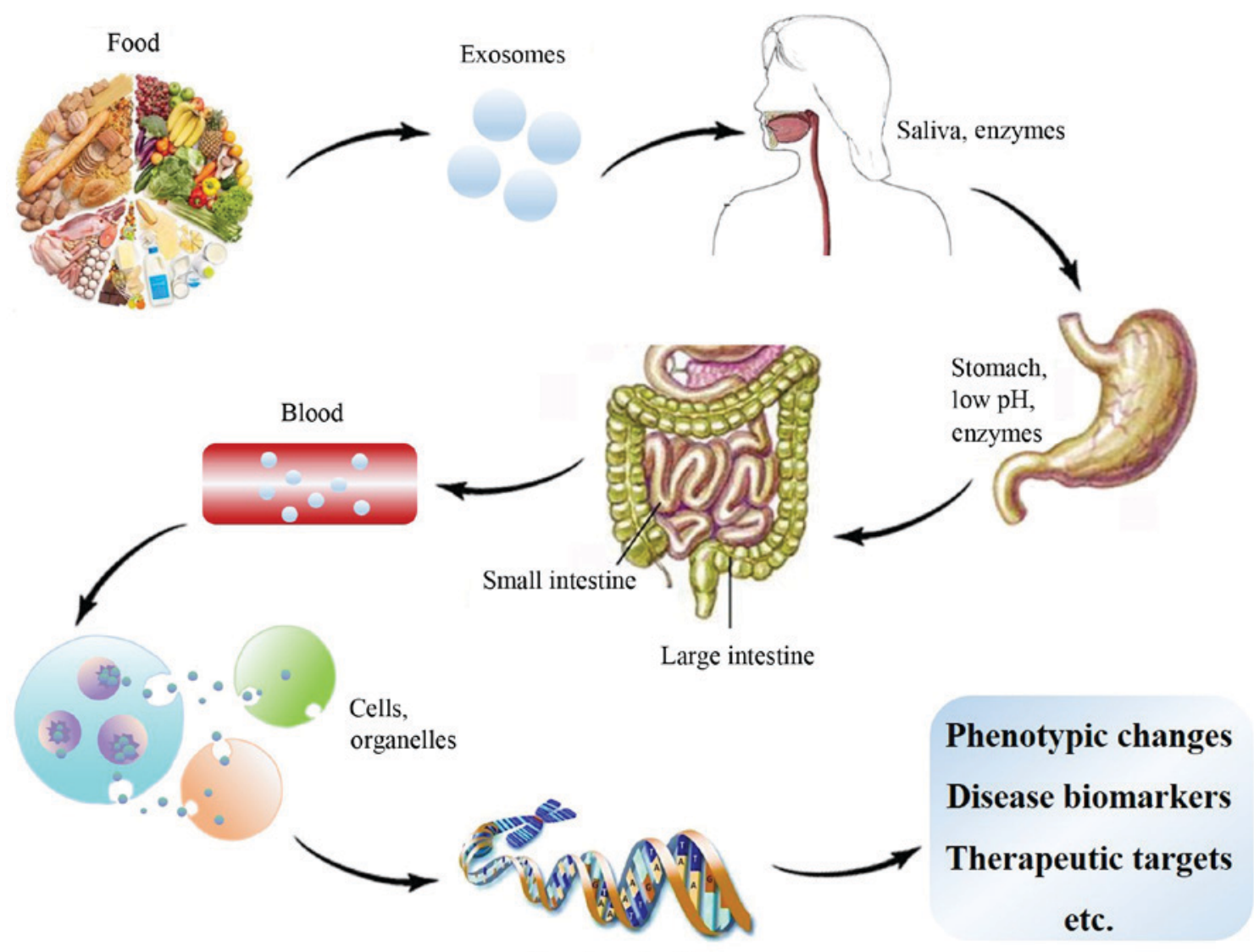

Genetic materials

Figure 2. Dietary exosomes are resistant to damage from digestion and function in target cells.

inhibit the PKA pathway, which then accordingly facilitates the differentiation of hMSCs into chondrocytes $(40,41)$.

$\mathrm{KBD}$ is characterized by upregulated apoptosis and necrosis of chondrocytes in the deep zone of articular cartilage (42). These pathological changes are usually combined with the increased expression of type I collagen and MMPs, as well as decreased productions of type II and type $\mathrm{X}$ collagens and degradation of aggrecan (43). A meta-analysis of 139 studies describing differentially expressed miRNAs in osteoarticular diseases indicated that miR-23b was overexpressed in lesion chondrocytes and cartilage tissue (44). Therefore, the pathological changes in KBD may be regulated by miR-23b and the PKA pathway.

\section{Dietary exosomal miRNAs are resistant to digestion and exert functions across species}

It has been confirmed that miRNAs are also contained in vegetables, meat and animal products, and insufficiency of these dietary sources of miRNAs cannot be compensated for by endogenous synthesis (22). The cargo of exogenous and endogenous exosomes is released and enters the circulation, which is then taken up by different organs. However, it is currently elusive how dietary exosomes are specifically targeted to the recipient cells (45). It has been proven that dietary miRNAs may be absorbed and utilized by mammalians as a form of exosomal cargo to regulate vital pathways and participate in responses to pathological triggers in the body to promote health, such as the tumor-suppressive effect of milk-derived exosomal miRNAs (46) and the role of grape-derived exosomal cargo (including miRNAs) in protecting mice from dextran sulfate sodium-induced colitis (47).

A simulation of the digestion process has indicated that the protection of the exosome, their cargos, including RNA, miRNAs and other degradable bioactive molecules, is able to resist damage caused by stomach conditions and still retains bioactivity in target cells, where they may be utilized (Fig. 2) (48). For instance, a computer-controlled gastrointestinal model of TNO intestinal model-1 proved that a large quantity of dairy milk Bos taurus (bta) miR-223 and bta-miR-125b withstood digestion under simulated gastrointestinal tract conditions. A large quantity of these 2 microRNAs was detected in the upper small intestine compartments, which supports their potential bioaccessibility (48). As another example, oral administration of exosomal curcumin in Sprague Dawley rats demonstrated 3-5 times higher levels in various organs compared with those achieved by administration of the free substance (49). miRNAs in a vegetarian diet, including soy milk and rice, are not significantly reduced by storage, processing and cooking. In addition, these dietary miRNAs maintain relatively high bioactivity after entering the digestive tract (50).

The results of previous studies suggest that exosomal cargo may stimulate cell growth and differentiation in vitro 


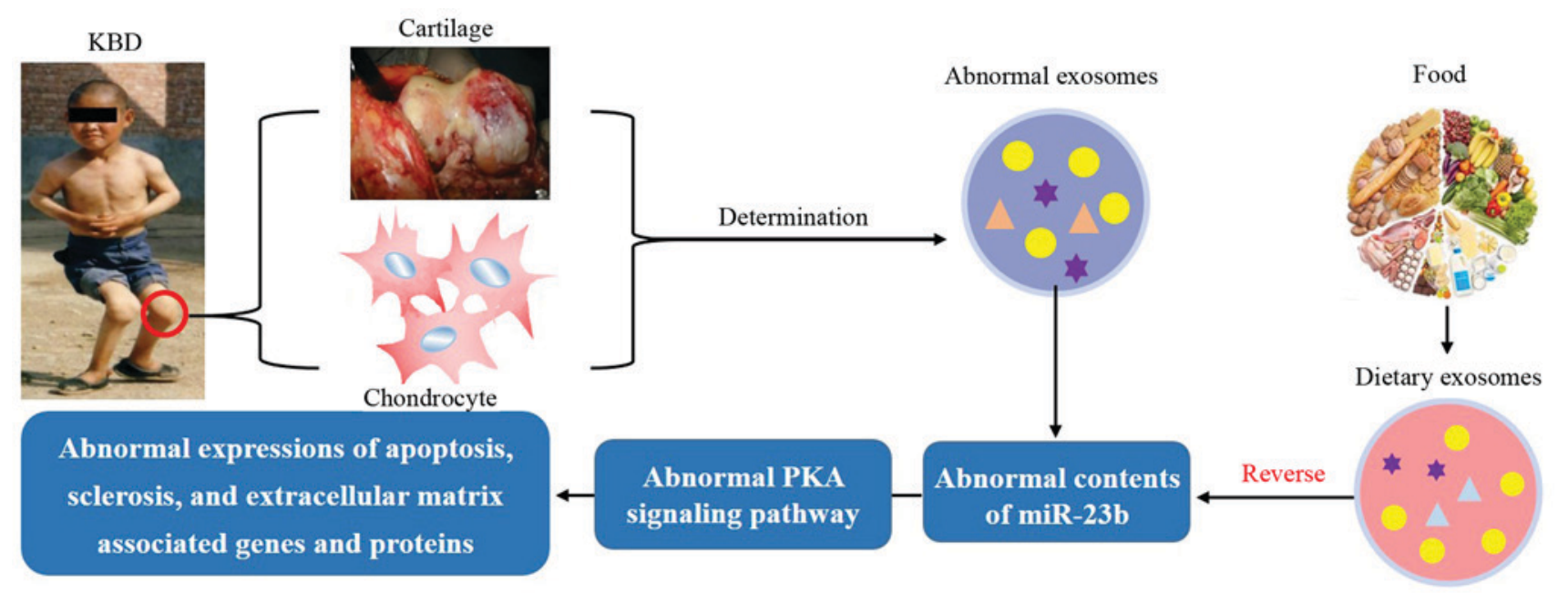

Figure 3. Proposal of study hypothesis: Dietary exosome reverses abnormal cargo contents in chondrocytes of patients with KBD. KBD, Kashin-Beck disease; miR, microRNA.

and may have therapeutic effects in in vivo models of disease. Exosomes derived from fruit and vegetables may be absorbed by macrophages and monocytes so that exosomal cargo may have a beneficial effect by inhibiting the inflammatory reaction $(51,52)$. Grape exosome-like nanoparticles added in culture media of stem cells facilitated the processes of proliferation and organ differentiation, and they also effectively elongated the life span of mice (47). Animal models of spontaneous polyarthritis and collagen-induced arthritis indicated that inflammatory regulation factor-associated miRNAs (miR-30a, -223 and -92a) in milk-derived exosomes may delay the occurrence of arthritis. This treatment was also effective in dose-dependently alleviating physical and pathological manifestations, including ankle swelling, cartilage damage and bone marrow inflammation. Furthermore, the treatment may decrease the serum levels of pro-inflammatory cytokines, including IL-6, growth-regulated alpha protein, monocyte chemoattractant protein-1 and anti-collagen immunoglobulin G2a, to facilitate the prevention and treatment of articular cartilage injury (53).

\section{Potential protective effect of dietary exosome-miR-23b in residents of endemic regions and KBD patients from articular cartilage injury stimulated by environmental risk factors through regulation of the PKA pathway}

The preliminary studies mentioned above encourage further investigation. There may be an abnormal quantity of exosome-miR-23b in chondrocytes affected by KBD, which may lead to the dysregulation of the PKA pathway and stimulate further pathological changes.

If this is the case, to reverse the pathological changes associated with KBD, intake of dietary (e.g., milk-derived) exosome-miR-23b should be able to positively regulate the PKA pathway to protect chondrocytes against damage induced by environmental risk factors, namely low selenium and T-2 toxin (Fig. 3).

Therefore, further study is warranted to i) clarify the association between exosome and the disease; ii) verify the interaction between miR-23b and the PKA pathway in KBD; and iii) identify whether dietary exosome-miR-23b inhibits articular cartilage injury stimulated by environmental risk factors through regulating the PKA pathway.

\section{Conclusions}

The pathogenesis of KBD, which is stimulated by environmental risk factors, is still a conundrum, and the protective mechanisms of dietary components in preventing KBD remains elusive. Supplementation of exosomal miRNA has been suggested as a promising therapeutic method to prevent osteoarticular diseases. miR-23b and the PKA pathway have been identified to be abnormal in cartilage tissue and chondrocytes of KBD patients. Hence, it is worth exploring whether dietary exosome-miR-23b inhibits articular cartilage injury stimulated by environmental risk factors through regulating the PKA pathway to prevent KBD.

\section{Acknowledgements}

Not applicable.

\section{Funding}

This study was financially supported by the China Postdoctoral Science Foundation (grant no. 2017M613153), the China Postdoctoral Science Foundation (grant no. 2017M623197), the National Key R\&D Program of China (grant no. 2016YFE0119100) and the Innovative Training Program Fund for College Students (201610698090).

\section{Availability of data and materials}

Not applicable.

\section{Authors' contributions}

$\mathrm{YN}, \mathrm{XW}$ and $\mathrm{XG}$ conceived the study and contributed in writing the manuscript. PZ, AL, XQ and ML aquired and analyzed the data. 


\section{Ethics approval and consent to participate}

Not applicable.

\section{Consent for publication}

Not applicable.

\section{Competing interests}

The authors have no competing interest to declare.

\section{References}

1. Huang Q, Zhou ZK, Ma J, Li Y, Yang X, Shen B, Yang J, Kang PD and Pei FX: The arthropathic and functional impairment features of adult Kashin-Beck disease patients in Aba Tibetan area in China. Osteoarthritis Cartilage 23: 601-606, 2015.

2. Yaqun K, Ren Pbc, Jun W, Pei Jcq, Jia Zwg and Ta Dzs: To investigate and analyze the condition of Kaschin-Beck disease around 7-12 years old children in Bianba county of Tibet in 2013. Chin J Infect Control 5: 350-351, 2014.

3. Lei R, Jiang N, Zhang Q, Hu S, Dennis BS, He S and Guo X: Prevalence of selenium, T-2 toxin and deoxynivalenol in kashin-beck disease areas in qinghai province, Northwest China. Biol Trace Elem Res 171: 34-40, 2016.

4. Wang J, Li H, Yang L, Li Y, Wei B, Yu J and Feng F: Distribution and translocation of selenium from soil to highland barley in the Tibetan plateau kashin-beck disease area. Environ Geochem Health 39: 221-229, 2017.

5. Dai X, Li Y, Zhang R, Kou Y, Mo X, Cao J and Xiong Y: Effects of sodium selenite on c-Jun $\mathrm{N}$-terminal kinase signalling pathway induced by oxidative stress in human chondrocytes and c-Jun $\mathrm{N}$-terminal kinase expression in patients with Kashin-Beck disease, an endemic osteoarthritis. Br J Nutr 115: 1547-1555, 2016.

6. Dermience M, Lognay G, Mathieu F and Goyens P: Effects of thirty elements on bone metabolism. J Trace Elem Med Biol 32: 86-106, 2015.

7. Dermience M, Mathieu F, Li XW, Vandevijvere S, Claus W, De Maertelaer V, Dufourny G, Bin L, Yangzom D and Lognay G: Minerals and trace elements intakes and food consumption patterns of young children living in rural areas of Tibet autonomous region, P.R. China: A cross-sectional survey. Healthcare (Basel) 5: pii: E12, 2017.

8. Wang X, Zhang Y, Chang Y, Duan D, Sun Z and Guo X Elevation of IGFBP2 contributes to mycotoxin T-2-induced chondrocyte injury and metabolism. Biochem Biophys Res Commun 478: 385-391, 2016.

9. Chen JH, Xue S, Li S, Wang ZL, Yang H, Wang W, Song D, Zhou $\mathrm{X}$ and Chen C: Oxidant damage in Kashin-Beck disease and a rat Kashin-Beck disease model by employing T-2 toxin treatment under selenium deficient conditions. J Orthop Res 30: 1229-1237, 2012.

10. Shi XW, Guo X, Ren FL, Lü AL and Zhang YZ: Familial aggregation and sibling heritability in Kashin-Beck disease. Nan Fang Yi Ke Da Xue Xue Bao 28: 1187-1189, 2008 (In Chinese).

11. Shi XW, Lv AL, Ren FL, Li WR, Kang LL and Guo X: Transmission disequilibrium test for 15 short tandem repeat loci in Kashin-Beck disease and their interaction with low selenium. Nan Fang Yi Ke Da Xue Xue Bao 31: 567-571, 2011 (In Chinese).

12. Guo X, Ma WJ, Zhang F, Ren FL, Qu CJ and Lammi MJ: Recent advances in the research of an endemic osteochondropathy in China: Kashin-Beck disease. Osteoarthritis Cartilage 22: 1774-1783, 2014

13. Yu FF, Han J, Wang X, Fang H, Liu H and Guo X: Salt-rich selenium for prevention and control children with Kashin-Beck disease: A meta-analysis of community-based trial. Biol Trace Elem Res 170: 25-32, 2016.

14. Sun LY, Li Q, Meng FG, Fu Y, Zhao ZJ and Wang LH: T-2 toxin contamination in grains and selenium concentration in drinking water and grains in Kaschin-Beck disease endemic areas of qinghai province. Biol Trace Elem Res 150: 371-375, 2012.
15. Zhou X, Wang Z, Chen J, Wang W, Song D, Li S, Yang H, Xue $S$ and Chen C: Increased levels of IL- 6 , IL-1 $\beta$, and TNF- $\alpha$ in Kashin-Beck disease and rats induced by T-2 toxin and selenium deficiency. Rheumatol Int 34: 995-1004, 2014

16. Ning YJ, Wang X, Wang S, Zhang F, Zhang L, Lei Y and Guo X: Is it the appropriate time to stop applying selenium enriched salt in Kashin-Beck disease areas in China? Nutrients 7: 6195-6212, 2015.

17. Ning YJ, Wang X, Ren L and Guo X: Effects of dietary factors on selenium levels of children to prevent Kashin-Beck disease during a high-prevalence period in an endemic area: A cohort study. Biol Trace Elem Res 153: 58-68, 2013

18. Yu FF, Liu H and Guo X: Integrative multivariate logistic regression analysis of risk factors for Kashin-Beck disease. Biol Trace Elem Res 174: 274-279, 2016.

19. Huang H, Li F, Yang X, et al: The Evaluation result analysis of control and elimination of kaschin-beck disease in 32 counties of sichuan province. J Prev Med Inf 33: 355-360, 2017.

20. Gong H-q, Zhao S-c, Ni M-c-j, Guo M and Li Q-w: Monitoring report of Kashin-Beck disease in Changdu Region of Tibet in 2014. Foreign Med Sci Section Med 36: 270-273, 2015.

21. Votteler J, Ogohara C, Yi S, Hsia Y, Nattermann U, Belnap DM, King NP and Sundquist WI: Designed proteins induce the formation of nanocage-containing extracellular vesicles. Nature 540: 292-295, 2016

22. Zempleni J, Aguilar-Lozano A, Sadri M, Sukreet S, Manca S, Wu D, Zhou F and Mutai E: Biological activities of extracellular vesicles and their cargos from bovine and human milk in humans and implications for infants. J Nutr 147: 3-10, 2017.

23. Yáñez-Mó M, Siljander PR, Andreu Z, Zavec AB, Borràs FE, Buzas EI, Buzas K, Casal E, Cappello F, Carvalho J, et al: Biological properties of extracellular vesicles and their physiological functions. J Extracell Vesicles 4: 27066, 2015.

24. Sun J, Aswath K, Schroeder SG, Lippolis JD, Reinhardt TA and Sonstegard TS: MicroRNA expression profiles of bovine milk exosomes in response to Staphylococcus aureus infection. BMC Genomics 16: 806, 2015.

25. Kato T, Miyaki S, Ishitobi H, Nakamura Y, Nakasa T, Lotz MK and Ochi M: Exosomes from IL-1 $\beta$ stimulated synovial fibroblasts induce osteoarthritic changes in articular chondrocytes. Arthritis Res Ther 16: R163, 2014.

26. Withrow J, Murphy C, Liu Y, Hunter M, Fulzele S and Hamrick MW: Extracellular vesicles in the pathogenesis of rheumatoid arthritis and osteoarthritis. Arthritis Res Ther 18: 286, 2016.

27. Chen J, Luo M, Wang W, Zhang Z, He Y, Duance VC, Hughes CE, Caterson B and Cao J: Altered proteolytic activity and expression of MMPs and aggrecanases and their inhibitors in Kashin-Beck disease. J Orthop Res 33: 47-55, 2015.

28. Tao SC, Yuan T, Zhang YL, Yin WJ, Guo SC and Zhang CQ: Exosomes derived from miR-140-5p-overexpressing human synovial mesenchymal stem cells enhance cartilage tissue regeneration and prevent osteoarthritis of the knee in a rat model. Theranostics 7: 180-195, 2017.

29. Wang Y, Yu D, Liu Z, Zhou F, Dai J, Wu B, Zhou J, Heng BC, Zou XH, Ouyang $\mathrm{H}$ and Liu $\mathrm{H}$ : Exosomes from embryonic mesenchymal stem cells alleviate osteoarthritis through balancing synthesis and degradation of cartilage extracellular matrix. Stem Cell Res Ther 8: 189, 2017.

30. Zhang S, Chu WC, Lai RC, Lim SK, Hui JH and Toh WS: Exosomes derived from human embryonic mesenchymal stem cells promote osteochondral regeneration. Osteoarthritis Cartilage 24: 2135-2140, 2016.

31. Toh WS, Lai RC, Hui JHP and Lim SK: MSC exosome as a cell-free MSC therapy for cartilage regeneration: Implications for osteoarthritis treatment. Semin Cell Dev Biol 67: 56-64, 2017.

32. Mao G, Zhang Z, Huang Z, Chen W, Huang G, Meng F, Zhang $Z$ and Kang Y: MicroRNA-92a-3p regulates the expression of cartilage-specific genes by directly targeting histone deacetylase 2 in chondrogenesis and degradation. Osteoarthritis Cartilage 25: 521-532, 2017.

33. Gabler J, Ruetze M, Kynast KL, Grossner T, Diederichs S and Richter W: Stage-specific miRs in chondrocyte maturation: Differentiation-dependent and hypertrophy-related miR clusters and the miR-181 family. Tissue Eng Part A 21: 2840-2851, 2015.

34. Liu H, Hao W, Wang X and Su H: miR-23b targets Smad 3 and ameliorates the LPS-inhibited osteogenic differentiation in preosteoblast MC3T3-E1 cells. J Toxicol Sci 41: 185-193, 2016. 
35. van der Harg JM, Eggels L, Bangel FN, Ruigrok SR, Zwart R, Hoozemans JJM, la Fleur SE and Scheper W: Insulin deficiency results in reversible protein kinase A activation and tau phosphorylation. Neurobiol Dis 103: 163-173, 2017.

36. Liu F, Xiao Y, Ji XL, Zhang KQ and Zou CG: The cAMP-PKA pathway-mediated fat mobilization is required for cold tolerance in C. elegans. Sci Rep 7: 638, 2017.

37. Zayed N, Afif H, Chabane N, Martel-Pelletier J, Pelletier JP and Fahmi H: Prostaglandin D2 inhibits interleukin-1-b-induced matrix metalloproteinase- 1 and -13 production by human chondrocytes via its DP1 receptor and cAMP/PKA pathway. Osteoarthritis Cartilage 16 (Suppl 4): S90-S91, 2008.

38. Zayed N, Afif H, Chabane N, Mfuna-Endam L, Benderdour M, Martel-Pelletier J, Pelletier JP, Motiani RK, Trebak M, Duval N and Fahmi H: Inhibition of interleukin-1beta-induced matrix metalloproteinases 1 and 13 production in human osteoarthritic chondrocytes by prostaglandin D2. Arthritis Rheum 58: 3530-3540, 2008.

39. Yokoyama K, Ikeya M, Umeda K, Oda H, Nodomi S, Nasu A, Matsumoto Y, Izawa K, Horigome K, Kusaka T, et al: Enhanced chondrogenesis of induced pluripotent stem cells from patients with neonatal-onset multisystem inflammatory disease occurs via the caspase 1-independent cAMP/protein kinase A/CREB pathway. Arthritis Rheumatol 67: 302-314, 2015.

40. Ham O, Lee CY, Song BW, Lee SY, Kim R, Park JH, Lee J, Seo HH, Lee CY, Chung YA, et al: Upregulation of miR-23b enhances the autologous therapeutic potential for degenerative arthritis by targeting PRKACB in synovial fluid-derived mesenchymal stem cells from patients. Mol Cells 37: 449-456, 2014.

41. Ham O, Song BW, Lee SY, Choi E, Cha MJ, Lee CY, Park JH, Kim IK, Chang W, Lim S, et al: The role of microRNA-23b in the differentiation of MSC into chondrocyte by targeting protein kinase A signaling. Biomaterials 33: 4500-4507, 2012.

42. Luo M, Chen J, Li S, Sun H, Zhang Z, Fu Q, Li J, Wang J, Hughes CE, Caterson B and Cao J: Changes in the metabolism of chondroitin sulfate glycosaminoglycans in articular cartilage from patients with Kashin-Beck disease. Osteoarthritis Cartilage 22: 986-995, 2014

43. Wang W, Guo X, Chen J, Xu P and Lammi MJ: Morphology and phenotype expression of types I, II, III, and X collagen and MMP-13 of chondrocytes cultured from articular cartilage of Kashin-Beck disease. J Rheumatol 35: 696-702, 2008.
44. Wang $X$, Ning Y, Zhou B, Yang L, Wang Y and Guo X Osteoarthritis associated microRNA expression signature: Integrated bioinformatics analysis. Mol Med Rep, 2017.

45. Guay C and Regazzi R: Exosomes as new players in metabolic organ cross-talk. Diabetes Obes Metab 19 (Suppl 1): S137-S146, 2017.

46. Otsuka K, Yamamoto Y, Matsuoka R and Ochiya T: Maintaining good miRNAs in the body keeps the doctor away?: Perspectives on the relationship between food-derived natural products and microRNAs in relation to exosomes/extracellular vesicles. Mol Nutr Food Res 62, 2018.

47. Ju S, Mu J, Dokland T, Zhuang X, Wang Q, Jiang H, Xiang X, Deng ZB, Wang B, Zhang L, et al: Grape exosome-like nanoparticles induce intestinal stem cells and protect mice from DSS-induced colitis. Mol Ther 21: 1345-1357, 2013.

48. Benmoussa A, Lee CH, Laffont B, Savard P, Laugier J, Boilard E, Gilbert C, Fliss I and Provost P: Commercial dairy cow milk microRNAs resist digestion under simulated gastrointestinal tract conditions. J Nutr 146: 2206-2215, 2016.

49. Aqil F, Munagala R, Jeyabalan J, Agrawal AK and Gupta R: Exosomes for the enhanced tissue bioavailability and efficacy of curcumin. AAPS J 19: 1691-1702, 2017.

50. Philip A, Ferro VA and Tate RJ: Determination of the potential bioavailability of plant microRNAs using a simulated human digestion process. Mol Nutr Food Res 59: 1962-1972, 2015.

51. Yarmarkovich $M$ and Hirschi KD: Digesting dietary miRNA therapeutics. Oncotarget 6: 13848-13849, 2015.

52. Yang J, Farmer LM, Agyekum AA and Hirschi KD: Detection of dietary plant-based small RNAs in animals. Cell Res 25: 517-520, 2015.

53. Arntz OJ, Pieters BC, Oliveira MC, Broeren MG, Bennink MB, de Vries M, van Lent PL, Koenders MI, van den Berg WB, van der Kraan PM and van de Loo FA: Oral administration of bovine milk derived extracellular vesicles attenuates arthritis in two mouse models. Mol Nutr Food Res 59: 1701-1712, 2015.

This work is licensed under a Creative Commons Attribution-NonCommercial-NoDerivatives 4.0 International (CC BY-NC-ND 4.0) License. 